\title{
Energy Landscapes of a Pair of Adsorbed Peptides
}

\author{
James A. Ross-Naylor†, Milan Mijajlovic $\ddagger$ and Mark J. Biggs§* \\ †School of Chemical Engineering, The University of Adelaide, Adelaide, South Australia, 5005 \\ Australia \\ ¥School of Engineering, Newcastle University, NE1 7RU, United Kingdom \\ §College of Science and Technology, Nottingham Trent University, Nottingham, NG11 8NS, \\ United Kingdom
}

*Corresponding author. Phone: +44 (0)115 848 3840. E-mail: mark.biggs@ntu.ac.uk.

The manuscript was written through contributions of all authors. All authors have given approval to the final version of the manuscript. 


\section{ABSTRACT}

The wide relevance of peptide adsorption in natural and synthetic contexts means it has attracted much attention. Molecular Dynamics (MD) simulation has been widely used in these endeavors. Much of this has focused on single peptides due to the computational effort required to capture the rare events that characterize their adsorption. This focus is, however, of limited practical relevance as in reality most systems of interest operate in the non-dilute regime where peptides will interact with other adsorbed peptides. As an alternative to MD simulation, we have used Energy Landscape Mapping (ELM) to investigate two met-enkephalin molecules adsorbed at a gas/graphite interface. Major conformations of the adsorbed peptides and the connecting transition states are elucidated along with the associated energy barriers and rates of exchange. The last of these makes clear that MD simulations are currently of limited use in probing the coadsorption of two peptides, let alone more. The constant volume heat capacity as a function of temperature is also presented. Overall, this study represents a significant step towards characterizing peptide adsorption beyond the dilute limit.

\section{INTRODUCTION}

Applications of peptide adsorption are widespread, including the formation, functionalization and impact of nanoparticles, ${ }^{1-6}$ biosensing, ${ }^{7-9}$ and biomedicine. ${ }^{10-15}$ Biomolecular adsorption is also of relevance to processes that occur in nature, and polypeptide segments of biomolecules are frequently used to study these processes, which include bodily reactions to foreign entities, ${ }^{16-17}$ cell adhesion, ${ }^{18}$ and the fibrillation of the amyloid-beta protein that is thought to have a role in Alzheimer's disease. ${ }^{19-21}$ This wide prevalence of peptide adsorption means it has attracted much study, both computational and experimental. ${ }^{22-25}$ Computational studies of peptide adsorption 
have yielded a significant degree of insight into the conformations formed by adsorbed peptides, and the key characteristics of adsorption processes. ${ }^{22-26}$ However, many such studies simulate only the adsorption of a single molecule, and adsorption studies that incorporate multiple peptides are typically limited to determining the adsorbed conformations on a short timescale. ${ }^{20,27}$ Since, in reality, peptide adsorption processes often involve multiple molecules adsorbing together, it is desirable to gain a deeper understanding of such systems.

Since processes involving adsorbed peptides typically occur over long time periods, ${ }^{28-29}$ required computational effort is a limiting factor in computational simulation of such processes. ${ }^{22,24}$ As the computational effort typically scales in a nonlinear way with the number of degrees of freedom, ${ }^{30}$ this factor becomes even more of an issue as the number of peptides increases. Coarse-graining models offer one way of addressing this issue, ${ }^{23}$ but much development and validation work still remains to be done before such models can be used to model peptide adsorption with confidence..$^{25}$

Energy landscape mapping (ELM) provides an alternative approach for computational study of atomic and molecular processes that is suited to probing the long timescales and rare events that typically characterize peptide adsorption. ${ }^{31-34}$ Although the exact details of its implementation vary, ELM may be generally defined as the use of mathematical algorithms to locate minima and saddle points on the potential energy surface (PES). A minimum corresponds to a stable conformation, while a first-order saddle point with steepest-descent paths leading to two minima represents a transition state between these minima. Constructing large-scale connected databases of these minima and saddle points can yield information about the underlying potential energy and free energy surfaces, and detailed path information and rates can be obtained through discrete path sampling (DPS). ${ }^{35}$ In recent work, the authors have demonstrated the applicability 
of ELM to a single adsorbed peptide. ${ }^{28,36}$ Here we demonstrate its application to two metenkephalin peptides at a gas/graphite interface. The models, methodologies and study details are outlined in the following section. Results are then presented and discussed, followed by the key conclusions and an outline of future areas of study.

\section{METHODOLOGY}

\subsection{MODEL}

In a previous study, ${ }^{36}$ the authors investigated a single met-enkephalin molecule above a flat, infinite graphite surface. This model is extended here to two met-enkephalin molecules (TyrGly-Gly-Phe-Met), modeled using the CHARMM36m force field. ${ }^{37}$ However, unlike the previous study in which the uncharged form was investigated due to its greater flexibility and thus interest, here the molecules were represented in the zwitterionic form (i.e. $\mathrm{NH}_{3}^{+}$and $\mathrm{COO}^{-}$ at the $\mathrm{N}$ - and C-termini, respectively), since it was presumed that the interactions of the charged termini would result in a more definite coupling of the peptides. A similar study on the uncharged form will be considered in future work. The CHARMM force field was not symmetrized, which may lead to a rougher PES/FES compared to the more correct symmetrized force field and some invalid stationary points corresponding to permutational isomers of the zwitterionic C-terminal residue; ${ }^{38}$ this was considered in the analyses of the PES and FES undertaken here.

Graphite was represented as two graphene layers, since it has been previously observed that layers beyond the first two have negligible interaction with adsorbing molecules. ${ }^{39}$ The Steele potential was used to model interactions between the peptide atoms and the surface. This potential is given by the following equation: ${ }^{40}$ 


$$
E_{S}=2 \pi \rho \sum_{j} \sum_{l=0}^{L-1} \varepsilon_{s j} \sigma_{s j}{ }^{2}\left[\frac{2}{5}\left(\frac{\sigma_{s j}}{z_{j}+l \Delta}\right)^{10}-\left(\frac{\sigma_{s j}}{z_{j}+l \Delta}\right)^{4}\right]
$$

where $l=0, \ldots, L-1$ is a counter over the layers of solid atoms in the surface up to the maximum, $L, \rho$ the density of atoms in each of the layers, $\Delta$ the distance between the layers, $z_{j}$ the distance from the surface of peptide atom $j$, and $\varepsilon_{s j}$ and $\sigma_{s j}$ the Lennard-Jones energy and length parameters, respectively. The Lennard-Jones parameters were calculated from the corresponding parameters for the surface, $\varepsilon_{s}$ and $\sigma_{s}$, and the atom, $\varepsilon_{j}$ and $\sigma_{j}$, using the LorentzBerthelot rules. The surface parameters are summarized in Table 1.

Table 1. Steele model parameters for graphite.

\begin{tabular}{|l|l|l|}
\hline parameter & value & reference \\
\hline$L$ & 2 & $a$ \\
\hline$\varepsilon_{s}$ & $0.05564 \mathrm{kcal} / \mathrm{mol}$ & $b$ \\
\hline$\sigma_{s}$ & $3.40 \AA$ & $b$ \\
\hline$\rho$ & 0.3807 atoms $/ \AA^{2}$ & $c$ \\
\hline$\Delta$ & $3.3555 \AA$ & $c$ \\
\hline
\end{tabular}

${ }^{a}$ Ref. 39

${ }^{b}$ Ref. 40

${ }^{c}$ Derived from literature bond length and unit cell height data. ${ }^{41}$

\subsection{METHODS}

The ELM procedure applied here is similar to that used in the authors' previous study. ${ }^{36}$ Firstly, a basin-hopping procedure with simulated annealing (SA-BH) was used to build an extensive database of local potential energy (PE) minima. Basin-hopping consists of a Monte Carlo simulation on a transformed PES, where every point is mapped to the locally minimized PE. This has the effect of eliminating the difficulties posed by PE barriers that are excessively 
high compared to the neighboring minima. ${ }^{42}$ Starting from a given structure, a random perturbation is applied followed by local PE minimization, and the Metropolis criterion is then applied to accept or reject the step. This procedure is repeated for a specified number of steps. The simulated annealing portion of the methodology involves gradually reducing the temperature used in application of the Metropolis criterion, allowing the algorithm to seek out lower-lying minima. In this study, the steps were taken by randomly perturbing the backbone dihedral angles of the met-enkephalin molecules up to a maximum step size, and minimization was carried out using the limited-memory Broyden-Fletcher-Goldfarb-Shanno (LBFGS) algorithm. ${ }^{43}$ A large number of SA-BH simulations were run independently in parallel, in order to quickly locate a large number of local minima and allow for the possibility that some simulations may converge to a region of the PES that does not contain the global minimum, which is a noted disadvantage of simulated annealing. ${ }^{42}$ Each simulation was initialized with a random structure and high starting step size for perturbations, ensuring effective randomization of the results.

Given the database of local minima obtained by SA-BH simulations, transition state searches were then applied to find saddle points connecting these minima, as well as additional minima that had not initially been found using SA-BH. Single-ended transition state searches were first used to probe the neighborhood of all known minima. In every such search, starting from a selected minimum, a small perturbation is applied, eigenvector following ${ }^{44}$ is used to locate a nearby saddle point, and the two minima adjoining this saddle point are located using the LBFGS algorithm. All newly found stationary points and their connectivity are recorded in the database.

When each known minimum had been used to seed a single-ended search a specified number of times, with additional searches conducted using low-lying minima within $5 \mathrm{kcal} / \mathrm{mol}$ of the global minimum, DPS ${ }^{35}$ techniques were then employed to continue searching for overlooked 
saddle points. Disconnectivity analysis ${ }^{45}$ was used to identify minima within $5 \mathrm{kcal} / \mathrm{mol}$ of the global minimum that were separated by barriers of more than $10 \mathrm{kcal} / \mathrm{mol}$, or disconnected from the global minimum entirely. DPS was then applied to all minima thus identified. For minima disconnected from the global minimum, an approach based on Dijkstra's shortest-path algorithm ${ }^{46}$ was used to search for connecting saddle points in order to establish a connected pathway of saddle points and minima. ${ }^{47}$ For pairs of low-lying minima connected to the global minimum but separated by a high PE barrier, a separate approach also based on Dijkstra's algorithm was used to determine the fastest transition path between the two endpoints, ${ }^{48}$ and double-ended transition state searches were applied between pairs of minima on this path, selected in order of increasing Euclidean separation. ${ }^{49}$ A double-ended transition state search involves the use of the doubly nudged elastic band (DNEB) method ${ }^{50}$ to identify saddle point candidates between the two endpoints, eigenvector following to precisely locate these saddle points, and the LBFGS algorithm to identify the connecting minima.

When attempts had been made to connect all low-lying minima in this way, significant welldefined structures were identified using disconnectivity analysis, and DPS was applied to refine the minimum energy pathway (MEP) between each pair of such structures. The MEP was calculated at each stage using the KSHORTESTPATHS algorithm, ${ }^{51}$ with rate constants estimated using harmonic densities of states. ${ }^{32,52}$ New saddle points and minima were found using the DPS procedure as previously stated, and also by applying the UNTRAP algorithm, ${ }^{53}$ which attempts to connect the endpoint structures with other minima near in configurational space but separated by high barriers. When both of these algorithms were unable to improve the MEP any further, the stationary point database was considered to be complete. 
To complement the PES computed directly from the database of PE stationary points, these stationary points were also used to derive the free energy (FE) stationary points, and thereby the free energy surface (FES), at specific temperatures. For each temperature, rate constants between PE minima connected by a saddle point were calculated using occupational probabilities derived from harmonic densities of states. ${ }^{32,52} \mathrm{PE}$ minima connected by rate constants above $10^{9} \mathrm{~s}^{-1}$ were combined to form new FE minima, and harmonic densities of states were then used to recalculate energies for these minima and associated transition states. ${ }^{54}$ This was done at $300 \mathrm{~K}$ and $350 \mathrm{~K}$ in order to illustrate how the nature of the FES varies with temperature. The harmonic approximation for densities of states was also used to calculate the constant volume heat capacity curve, as a summation over every PE minimum. ${ }^{55}$

This procedure was implemented using the software of Wales and co-workers, which is freely available on their website. ${ }^{56}$ This software was interfaced with the CHARMM program for PE calculations, ${ }^{57}$ which was modified to include the Steele potential. Molecular structure images used in this publication were generated using Visual Molecular Dynamics. ${ }^{58}$

\subsection{STUDY DETAILS}

A total of 100 SA-BH simulations were conducted, each of 200,000 steps. A temperature of $k_{B} T=5.0 \mathrm{kcal} / \mathrm{mol}$ was used for the first step, and this was decremented by $2 \times 10^{-3} \%$ after each step. The maximum step size for each dihedral angle was initially set to $360^{\circ}$, simulating complete randomization of the secondary structure. This was adjusted within the range of $(0$, $360^{\circ}$ ] every 50 steps, being increased by 5\% if the Metropolis acceptance ratio for the preceding 50 steps was greater than 0.5 , and decreased by $5 \%$ otherwise. Starting points for single-ended transition state searches were produced by applying random deviations of up to $0.01 \AA$ in each Cartesian coordinate from the local PE minima. The DNEB method was applied with 10 images 
and a maximum of 300 iterations. All applications of the LBFGS algorithm used a memory of the last 4 iterations, a maximum step size of $0.4 \AA$, initial guesses for the diagonals of the Hessian matrix of $0.1 \mathrm{kcal}^{2} / \mathrm{mol}^{2} \AA^{2}$, and a convergence criterion of the RMS gradient not exceeding $10^{-7} \mathrm{kcal} / \mathrm{mol} \AA$. 50 single-ended transition state searches were attempted from each local minimum within $5 \mathrm{kcal} / \mathrm{mol}$ of the global minimum, and 5 searches were attempted from each local minimum above this threshold.

\section{RESULTS AND DISCUSSION}

\subsection{ELM RESULTS AND VALIDITY}

ELM was successful in locating a total of 1,223,837 minima and 1,116,410 saddle points. These numbers compare favorably with studies of comparable size and complexity, ${ }^{28,36,59-62}$ which typically report total numbers of stationary points in the order of $10^{4}$ to $10^{6}$. This database of stationary points will not be exhaustive owing to the complexity of the system: complete mapping of even a single adsorbed molecule has been noted as impractical, ${ }^{63}$ and the presence of a second molecule with additional degrees of freedom further exacerbates this, since the number of stationary points scales exponentially with the number of degrees of freedom. ${ }^{32}$ However, based on our previous study where we obtained good agreement between ELM and replica exchange molecular dynamics simulation for a single adsorbed peptide, ${ }^{36}$ we believe the application of ELM here has identified the lowest PE minimum and probed that part of the PE surface connected to it that is likely to have the greatest impact on the dynamics of the system. ${ }^{32}$ The latter is in particular supported by Figure 1, which shows that the number of minima discovered within $5 \mathrm{kcal} / \mathrm{mol}$ of the global minimum plateaus over the course of the PES exploration, notwithstanding a sharp spike after about 1,160,000 minima that corresponds to the beginning of the DPS phase of the exploration. Of 8,674 minima below the $5 \mathrm{kcal} / \mathrm{mol}$ threshold, 
8,279 were connected to the global minimum by a PE barrier of less than $20 \mathrm{kcal} / \mathrm{mol}$, indicating considerable success in identifying paths between low-lying minima.

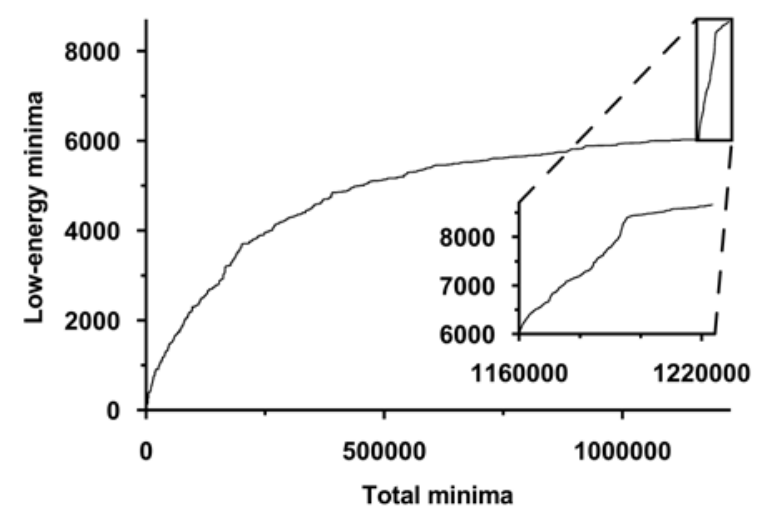

Figure 1. Variation of the number of minima found within $5 \mathrm{kcal} / \mathrm{mol}$ of the global minimum over the course of the exploration of the potential energy surface, which is defined in terms of the total number of minima found. Inset shows the additional minima found through the DPS analysis.

\subsection{STRUCTURES AND ENERGY LANDSCAPES}

Disconnectivity analysis on the database of stationary points resulted in the identification of three key conformations, each corresponding to a PE minimum at or near the global minimum PE and lower than any other minimum in its neighborhood on the PES. One of these structures is the global minimum, denoted as A, while the other two are denoted as B and C. Figure 2 displays these structures as viewed from the top (above the surface) and from the side. By inspection, it is clear that they are not permutational isomers and hence unrelated to the lack of symmetrization of the force field. All three structures share a common motif of the two met-enkephalin molecules lying anti-parallel on the surface, with the backbones interacting with one another and the Tyr1, Phe4 and Met5 sidechains extending out in opposite directions. A and B are almost 
identical, with the backbones binding strongly through hydrogen bonds between the $\mathrm{N}$-terminal $\mathrm{NH}_{3}{ }^{+}$group in one molecule and the Glys carboxyl group in the other. The only discernible difference between A and B occurs in one of the Met5 sidechains (left of the image), which adheres to the surface in A but peels upwards in B, interacting with the peptide backbone. C has a more distinct structure, with both molecules having arched backbones and the charged $\mathrm{N}$ - and C-termini all interacting with one another close to the surface. A random sampling of other stationary points found by ELM verified that structures lacking the anti-parallel motif observed here were discovered, but every such structure possessed a PE more than $20 \mathrm{kcal} / \mathrm{mol}$ higher than the global minimum. It can be concluded that all conformations reasonably contributing to the dynamics of the system possess this anti-parallel motif. 


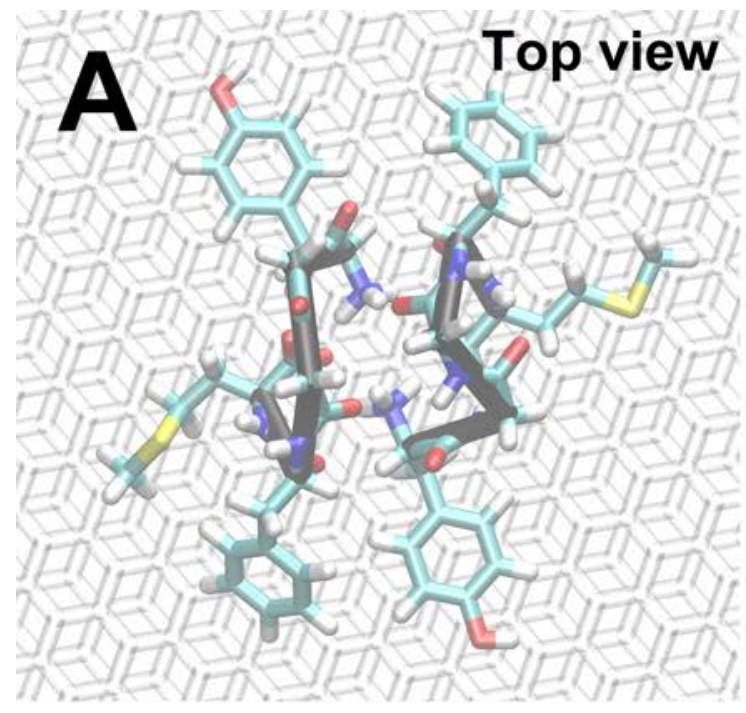

Side view

(from bottom end of top view)

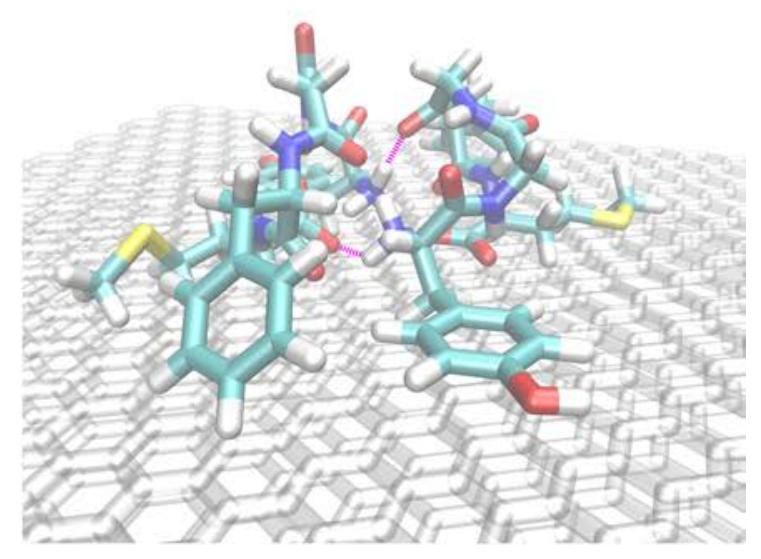

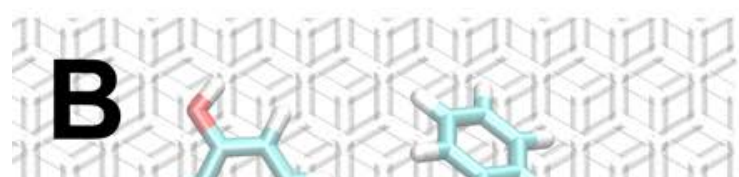

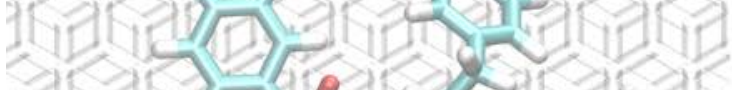
11 10 in 115010 ba 15 ID 3 id in is

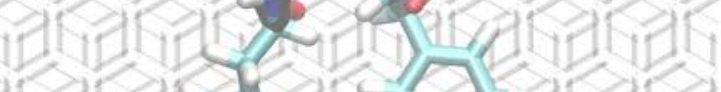

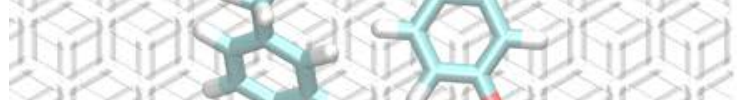
15 15 id

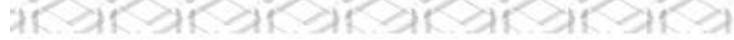
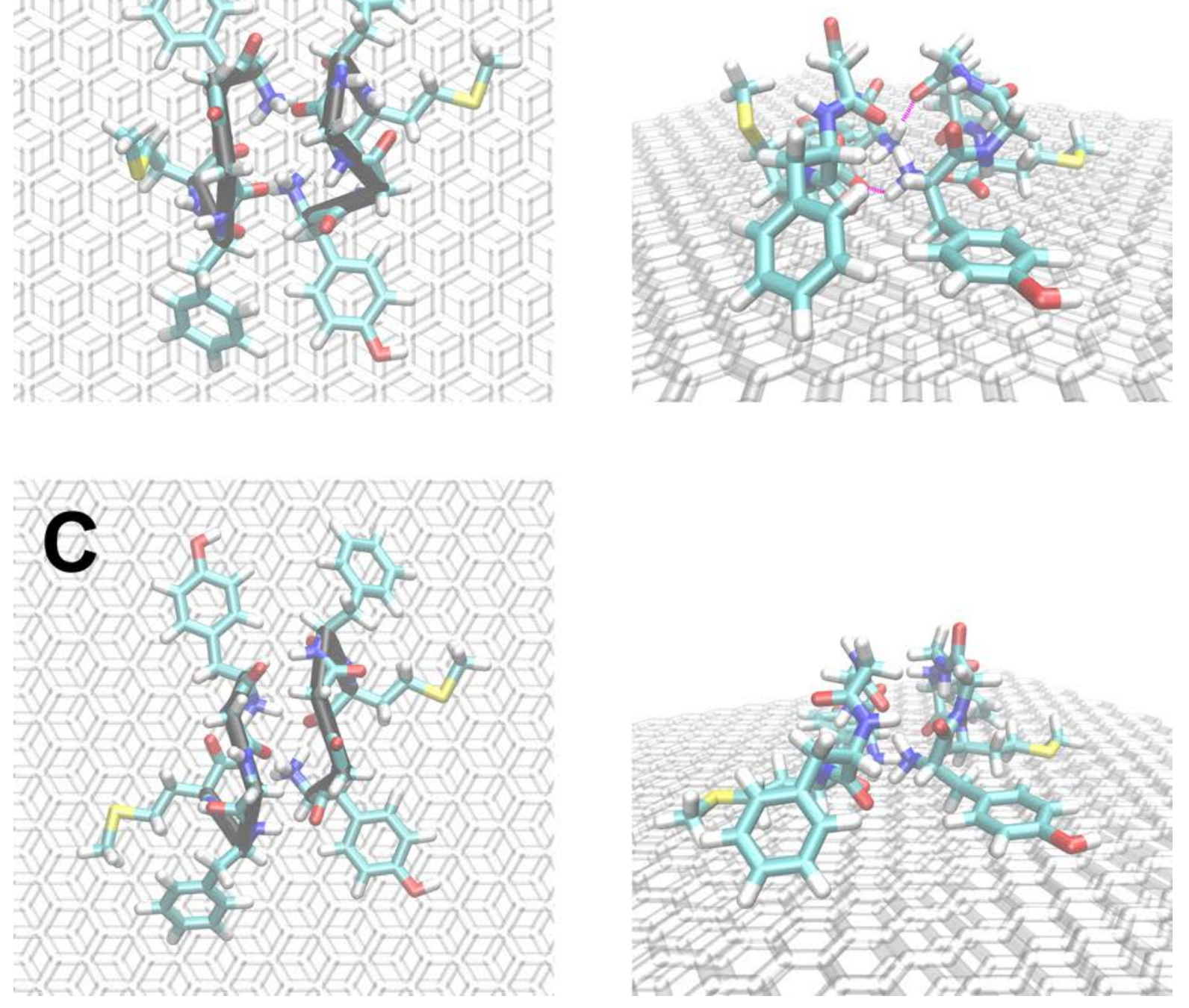
Figure 2. Global PE minimum structure (A) and the two other key local PE minimum structures (B and C) discovered for two zwitterionic met-enkephalin molecules adsorbed at the gas/graphite interface. The peptide backbones are represented on the leftmost images by black tubes connecting the $\mathrm{C}_{\alpha}$ atoms of each residue. Pink dashed lines on the rightmost images denote hydrogen bonds referred to in the text.

Figure 3 shows these three key structures, along with all the lowest connected stationary points, in a disconnectivity graph. A disconnectivity graph represents an underlying energy landscape as a connected tree, with termini corresponding to energy minima, and nodes corresponding to the energy barrier between any pair of minima. ${ }^{45}$ Compared to the corresponding PES obtained in our previous study of a single met-enkephalin molecule with uncharged termini adsorbed at the gas/graphite interface, ${ }^{36}$ the 'palm tree' motifs leading down to A, B and C suggests a PES with more defined structures; this is likely to be due to the presence of the charged termini in this study. The palm tree motif represents a funnel in the PES that energetically drives the conformation towards the minimum PE structure, and multiple such funnels indicate competing structures. ${ }^{64}$ In this case, the regions of the PES containing structures A and B are separated by a PE barrier of approximately $10 \mathrm{kcal} / \mathrm{mol}$, while the more distinct structure C is separated by a barrier of over $15 \mathrm{kcal} / \mathrm{mol}$. These PE barriers are very sizeable given the qualitative similarity of the conformations. As elucidated by the transition paths presented and discussed in the next section, it is likely that these high barriers are caused by the geometric complexity of the system, which poses a significant energetic challenge to even minor transitions. Each of the funnels associated with structures A, B and C possesses four low PE minima corresponding to the same structure; these are a consequence of the broken symmetry of 
the CHARMM force field. The lowest PE minimum corresponding to each structure was chosen for transition analysis.

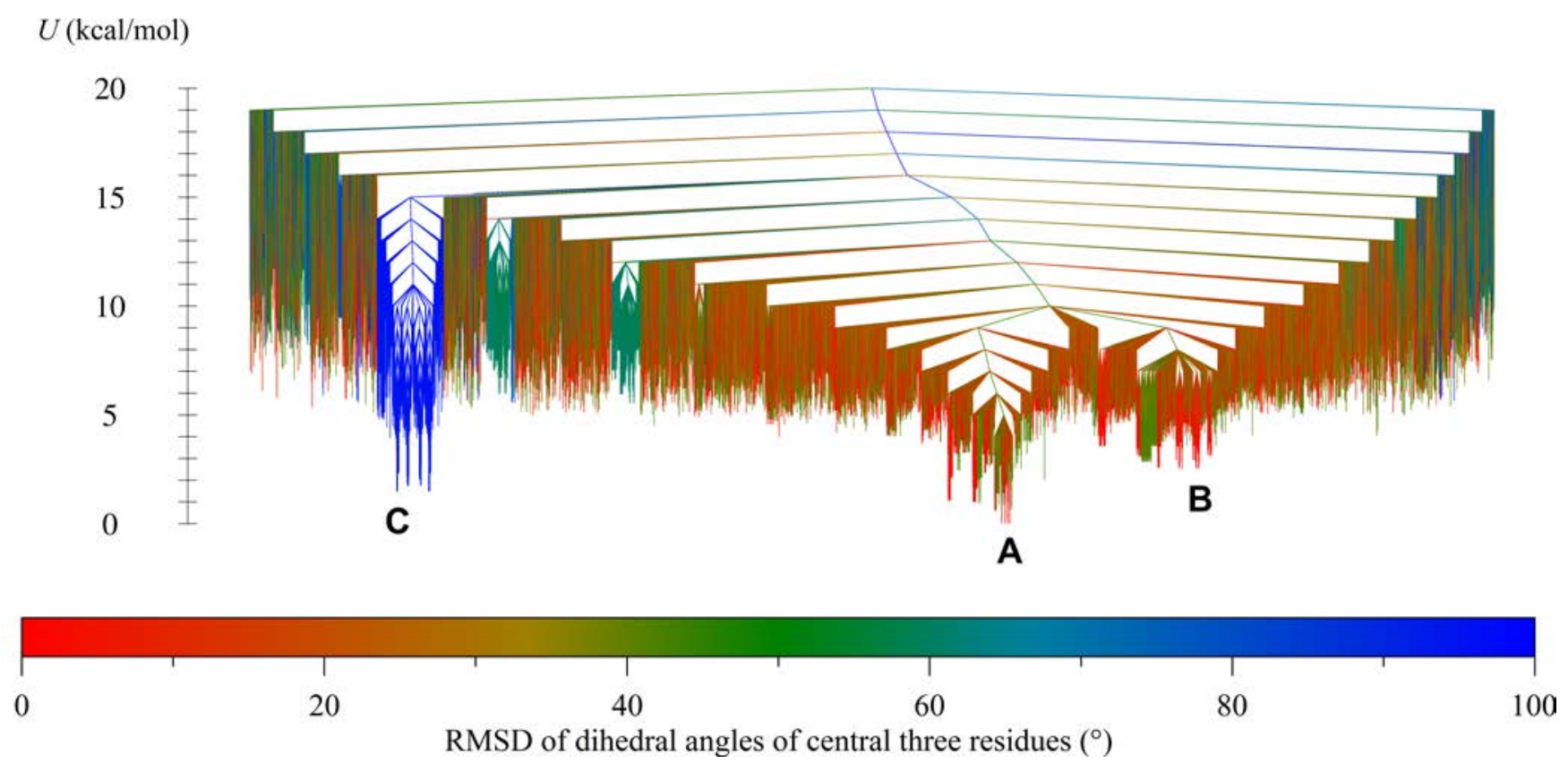

Figure 3. PE disconnectivity graph for two zwitterionic met-enkephalin molecules adsorbed at the gas/graphite interface. All minima connected to the global minimum by a maximum PE saddle point below $U=20 \mathrm{kcal} / \mathrm{mol}$ are shown. The labels $\mathrm{A}, \mathrm{B}$ and $\mathrm{C}$ correspond to the structures shown in Figure 2. Minima are colored according to the RMSD of the dihedral angles of the central three resides from the global minimum, using the displayed scale.

The color scale on Figure 3 provides further insight into the structure of the PES. Termini and connecting nodes are colored according to the root mean standard deviation of the six central backbone dihedral angles ( $\varphi_{2}, \psi_{2}, \varphi_{3}, \psi_{3}, \varphi_{4}$ and $\left.\psi_{4}\right)$ of both molecules, relative to the global minimum. This was chosen as an order parameter in light of past studies, which have shown the central three residues of met-enkephalin contribute most significantly to its conformation. ${ }^{65-66}$ This order parameter failed to distinguish between structures A and B, which is unsurprising 
given the only qualitative difference between the two structures occurs in the Mets sidechain. In fact, the variations in color from red to green in both funnels indicate that modest deviations in the peptide backbones prove more accessible than movement of the sidechain, which seems to play a significant role in stabilizing the conformation. The parameter is more successful in distinguishing structure $\mathrm{C}$, and other individual minima and minor funnels that are separated from the global minimum by high PE barriers. Other order parameters, such as the RMSD of atom positions and the number of peptide-peptide contacts, also failed to separate A from B and in some cases could not distinguish between any of the three (results not shown). This demonstrates that in systems where peptides and biomolecules are adsorbing in contact with one another, properties and functional groups that would not normally be considered when assessing the configurational space of a single molecule may become highly relevant, such as the Met5 sidechain in this case.

The disconnectivity graphs in Figure 4 display the FES at temperatures of $300 \mathrm{~K}$ and $350 \mathrm{~K}$, and complement the PES by showing the temperature dependence of the system. At both temperatures, structure A remains the global minimum, while $\mathrm{B}$ and $\mathrm{C}$ remain the only significant competing minima. It is notable that although the PE of structure $\mathrm{C}$ is lower than that of structure B, the reverse is true of their FE at $300 \mathrm{~K}$. This is intuitively due to the effect of entropy: as Figure 3 indicates, the region of configurational space corresponding to B contains more stationary points than $\mathrm{C}$ and is likely larger, permitting a greater degree of movement and perturbation within the molecules. The same effect is evident at $350 \mathrm{~K}$, at which point the amalgamation of minima connected by rate constants above $10^{9} \mathrm{~s}^{-1}$ causes structures A and B to merge. The FE of structure $\mathrm{C}$ relative to the global minimum holds steady at approximately $4 k_{B} T$, 
while the FE barrier decreases relative to the temperature, indicating, unsurprisingly, that the transition between structures $\mathrm{A} / \mathrm{B}$ and $\mathrm{C}$ becomes easier as the temperature is increased.

(a)

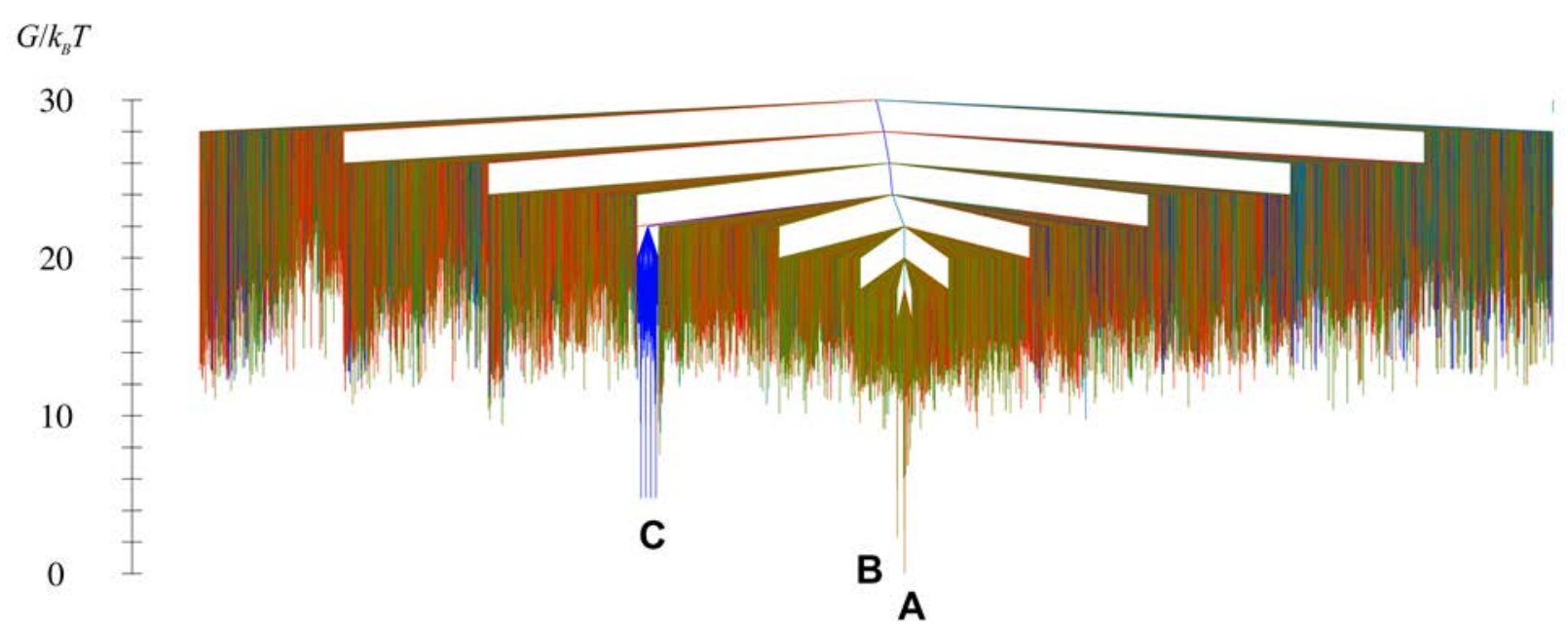

(b)
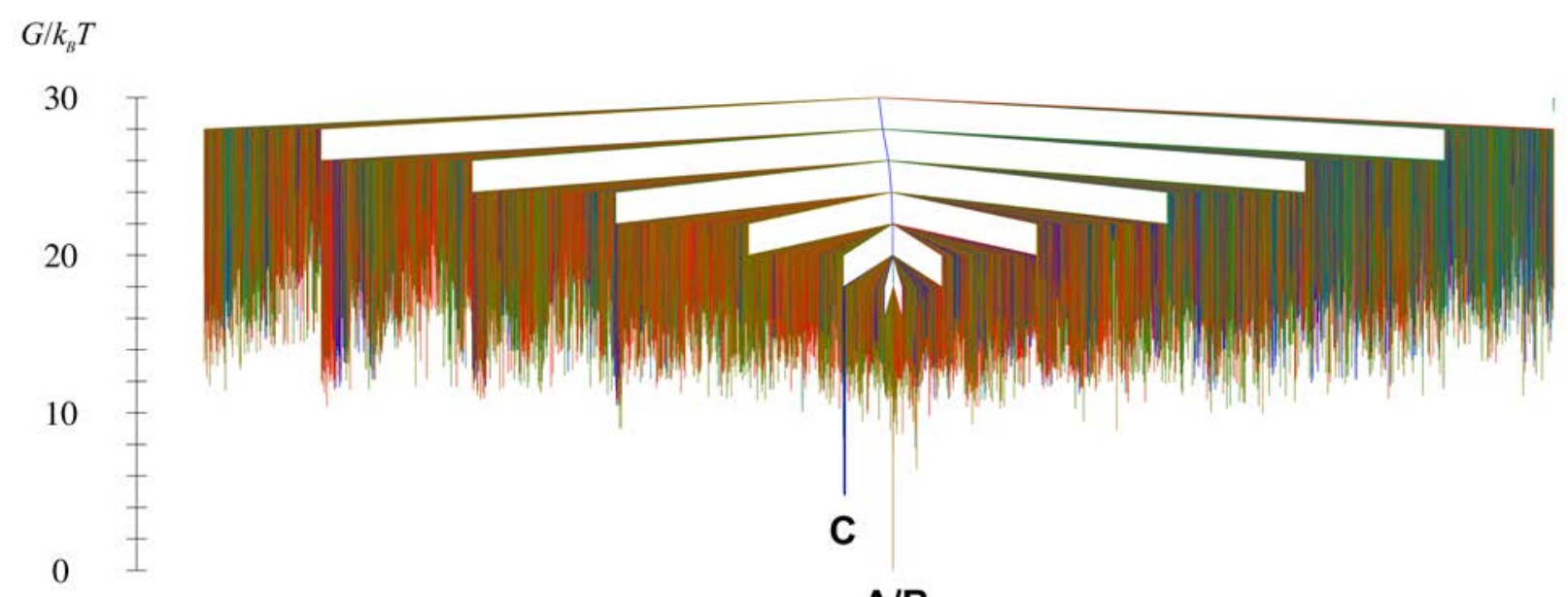

A/B

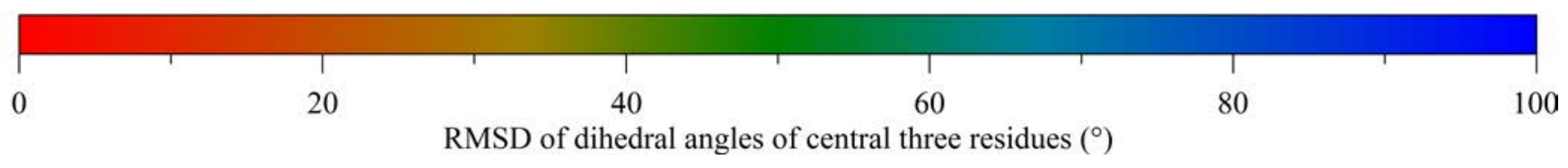

Figure 4. FE disconnectivity graphs for two zwitterionic met-enkephalin molecules adsorbed at the gas/graphite interface at temperatures at: (a) $300 \mathrm{~K}$; and (b) $350 \mathrm{~K}$. All minima connected to the global minimum by a maximum PE saddle point below $G=30 k_{B} T$ are shown. The labels A, $\mathrm{B}$ and $\mathrm{C}$ correspond to the structures shown in Figure 2. Minima are colored according to the 
RMSD of the dihedral angles of the central three resides from the global minimum, using the displayed scale.

Figure 5 displays the heat capacity of the system, calculated as a function of temperature. This may be compared with the heat capacity obtained for a single uncharged met-enkephalin molecule at a gas/graphite interface. ${ }^{36}$ Here, the heat capacity curve has one solitary peak, and its height, indicated by the scale of the $y$-axis, is quite small, indicating only a minor variance with temperature. This is consistent with the highly structured energy landscape of the two adsorbed peptides, as shown in Figure 3 and Figure 4, which contrasts with the rougher landscape of the single molecule. ${ }^{36}$ A point of inflection is observable on the heat capacity curve, at approximately $200 \mathrm{~K}$. This could indicate that the curve comprises two overlapping peaks: a minor peak at approximately $150 \mathrm{~K}$ and a major one at about $300 \mathrm{~K}$. These peaks, intuitively, are likely to correspond to the low-energy transition between A and B and the high-energy transition between $\mathrm{A} / \mathrm{B}$ and $\mathrm{C}$, respectively. It is worth noting that the harmonic approximation used in the calculation of heat capacities in this work may lead to inaccuracies, particularly at higher temperatures. ${ }^{55}$ However, comparison with simulation methods for computing the heat capacities of adsorbed peptides showed that the harmonic approximation was sufficient to replicate the qualitative features of the heat capacity curve, despite quantitative errors. ${ }^{36}$ 


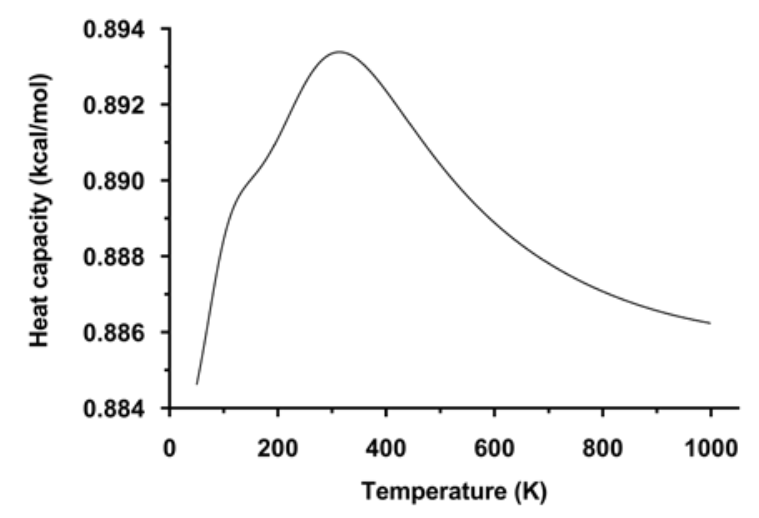

Figure 5. Constant volume heat capacity, as a function of temperature, calculated from the stationary point database obtained from ELM.

\subsection{TRANSITION PATHWAYS}

A more complete understanding of the key structures formed by the system is gained by considering the energy profile of the MEP between each pair of structures, and the conformations formed along this pathway. Figure 6 illustrates the paths between all three pairs of structures ( $A \leftrightarrow B, A \leftrightarrow C$ and $B \leftrightarrow C$ ). It should be noted that the $A \leftrightarrow B$ path is far shorter than the other two, which is to be expected given the structures' similarity. Also, the $A \leftrightarrow C$ and $B \leftrightarrow C$ paths share much in common, with both A and B transitioning to a meta-stable intermediate, denoted X, from which the full transition to C proceeds.

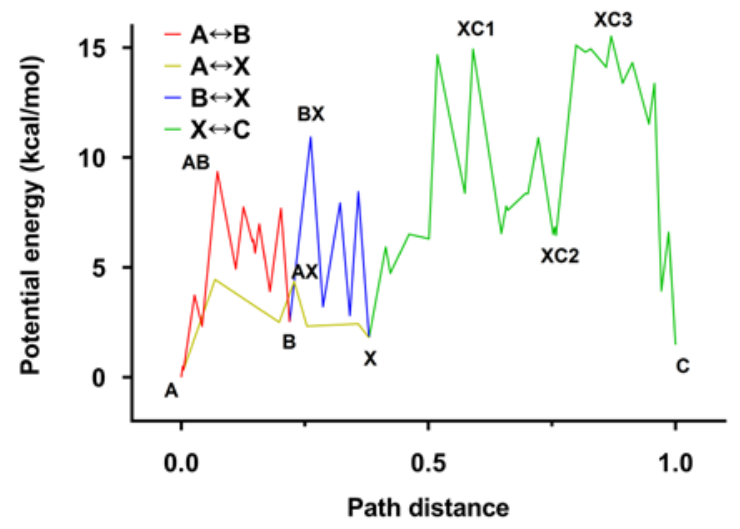


Figure 6. Variation of the PE along the MEPs between structures A, B and C shown in Figure 2, including intermediate structures labeled on this graph and displayed in Figure 7. The reference PE is that of the global minimum structure, A. The path distance is the minimized Euclidean distance between neighboring stationary points, scaled to the path between A and C. Lines between stationary points are provided as a guide to the eye only.

Major structures and intermediates highlighted in Figure 6 are illustrated in Figure 7, in the form of a flow chart showing the transitions. In general, these transitions consist of a series of structural changes that impact the peptides' interaction sites with one another and with the surface to a significant degree. This is presumably responsible for the high energy barriers present at a number of points along the transition paths, and hence the multi-funnel nature of the energy landscapes as illustrated in Figure 3 and Figure 4 . The primary motif in the $A \leftrightarrow B$ transition, and indeed that from $\mathrm{B}$ to $\mathrm{X}$ as well, is the rotation of the left-hand (as viewed) Met5 sidechain, between lying flat on the surface and peeling away to interact with the peptide backbone. Only in the final stages of the transition from B to $\mathrm{X}$ is there significant movement elsewhere, with the Gly2 residue on the left-hand molecule twisting parallel to the surface. The transition from A to $\mathrm{X}$, meanwhile, involves no change to the sidechain, and a PE barrier approximately half that of the $\mathrm{A} \leftrightarrow \mathrm{B}$ and $\mathrm{B} \leftrightarrow \mathrm{X}$ transitions. This further supports the hypothesis of the Met5 sidechains stabilizing the system. 


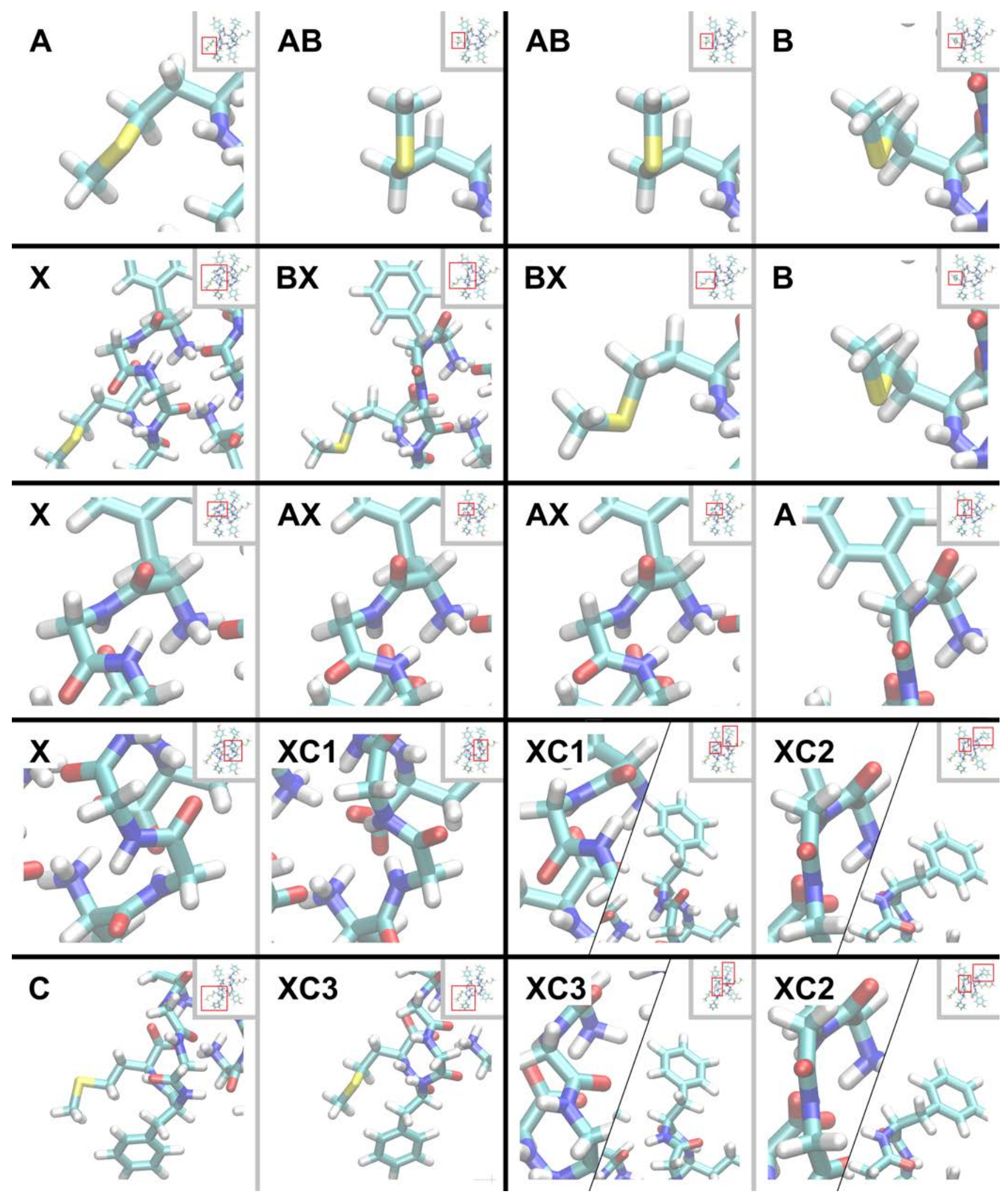


Figure 7. Drawings of transitions between structures A, B and C shown in Figure 2, and intermediates highlighted in Figure 6. The main images are zoomed in on the transitioning functional groups. Full structures are shown in insets.

The transition path between $\mathrm{X}$ and $\mathrm{C}$ takes place in two general stages, each with approximately equal PE barriers. Starting from X, firstly, both molecules’ backbones straighten and lengthen via the Gly2 residues rotating to arch over the surface, causing the Tyr1 and Phe 4 sidechains to peel away from each other. Meanwhile, the Glyз residue of the right-hand molecule rotates 180 degrees, breaking the hydrogen bond with the N-terminus of the left-hand molecule. The combined effect instead brings this N-terminus into closer contact with the C-termini of both molecules, resulting in a moderately stable intermediate, XC2. Following this, both the Gly2 and Glyз residues of the left-hand molecules rotate similarly, breaking the other Glys-N-terminus hydrogen bond and instead facilitating interactions between all four termini. At the final step, the left-hand Met5 sidechain rotates to lie flat on the surface, forming the final C structure.

The energy profiles displayed in Figure 6 give a general comparison of the energetic favorability of different paths and stages, but quantifying the timescales of the transitions requires the calculation of rate constants. Figure 8 displays rate constants for each of the reversible transitions between $\mathrm{A}, \mathrm{B}$ and $\mathrm{C}$ as a function of temperature, taking entropic effects into consideration. As the energy barrier would suggest, A and B interconvert relatively readily at $300 \mathrm{~K}$; at this temperature, the favored transition from B to A possesses a rate constant of approximately $10^{7} \mathrm{~s}^{-1}$, corresponding to a mean transition time of $100 \mathrm{~ns}$. Transitions to and from $\mathrm{C}$ are far slower, requiring times in the millisecond range at $300 \mathrm{~K}$, which is likely to be out of reach of most standard MD simulations, and in the microseconds at $450 \mathrm{~K}$. The narrowing gap between the forward and backward reactions for $A \leftrightarrow B$ and $A \leftrightarrow C$ with increasing temperature 
indicate that alternate structures to the global minimum A may be found in greater quantities at higher temperature. The $\mathrm{B} \leftrightarrow \mathrm{C}$ transition does not follow this trend: the rate constants are approximately equal at $250 \mathrm{~K}$, and the transition from C to B is favored thereafter; this likely arises due to structure B's entropic favorability overcoming its marginally higher PE with increasing temperature. In practice, it can be concluded that, due to the prevalence of $A$, the $\mathrm{A} \leftrightarrow \mathrm{B}$ and $\mathrm{A} \leftrightarrow \mathrm{C}$ transitions would dominate at all the surveyed temperatures. It should be noted that these rate constants consider only the single most favored path and ignore other less significant paths that would nonetheless contribute to the dynamics of the system.

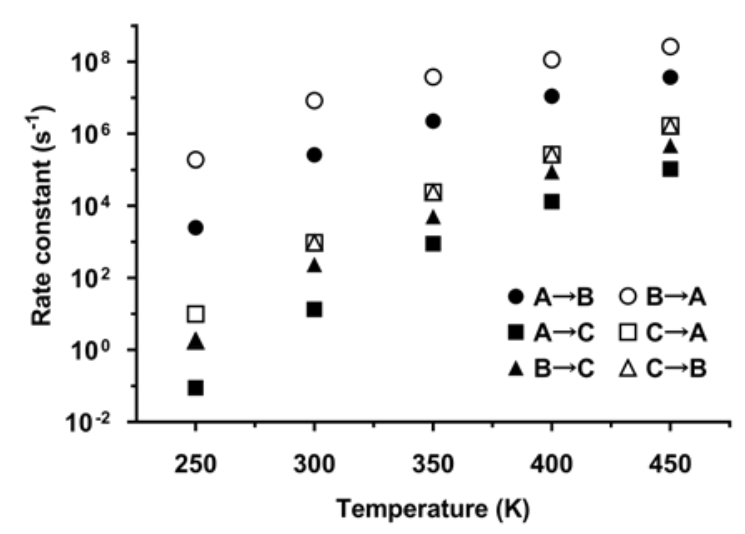

Figure 8. Variation of the rate constants with temperature for the transition paths between structures A, B and C as shown in Figure 6.

\section{CONCLUSIONS}

The conformations and energy landscapes of two zwitterionic met-enkephalin molecules at a gas/graphite interface were investigated using ELM. It was found that the system favors structures where the two peptides lie antiparallel on the surface with the backbones in close proximity and interacting through hydrogen bonds. Major conformations are distinguished by the orientation of the Met5 sidechains to the surface, and the functional groups to which the $\mathrm{N}$ - 
termini hydrogen-bond. Although these constitute only minor structural changes, the geometry of the system, involving two molecules in close proximity and the presence of a rigid surface, makes transitions between them relatively difficult and complex, occurring up to the millisecond range at $300 \mathrm{~K}$ in some cases. Both the specific conformations taken by two adsorbed metenkephalin molecules, and the ease of transitions between them, differed appreciably from a prior study of a single adsorbed met-enkephalin molecule at the gas/graphite interface. ${ }^{36}$ This has significant implications for studies of peptide adsorption: it is evident that results obtained from studies of a single adsorbed molecule will be of limited applicability to adsorption in systems that are not dilute.

The present work characterized a system similar to those benchmarked in prior studies. ${ }^{36,67}$ Future applications of ELM to multiple adsorbed peptides may consider peptides with aggregation properties, such as beta-sheet and helix formers. In general, however, further work is needed to validate and improve the applicability of ELM to biomolecule-surface interactions. Since the computational feasibility of ELM depends significantly on the number of degrees of freedom, ${ }^{32}$ modeling macromolecules such as proteins and simulating aqueous environments using explicit solvent presents difficulties. Efforts are ongoing to produce all-atom force fields compatible with liquid/solid interfacial systems $^{29}$ and reliable coarse-grained models for proteins. ${ }^{26}$ Finally, while the present work investigating the adsorption of two molecules on a surface represents a step towards modeling bulk adsorption, greater insights would be achieved by considering a larger number of molecules, and the development of a generalized strategy to this end would be desirable.

\section{ACKNOWLEDGMENT}


J.A.R. is grateful to the University of Adelaide for the receipt of an Australian Postgraduate Award. The support of the Australian Research Council Discovery Program (DP130101714) is gratefully acknowledged. Computational resources were provided by the Phoenix HPC service at the University of Adelaide.

\section{REFERENCES}

1. Slocik, J. M.; Naik, R. R. Sequenced Defined Biomolecules for Nanomaterial Synthesis, Functionalization, and Assembly. Curr. Opin. Biotechnol. 2017, 46, 7-13.

2. Walsh, T. R.; Knecht, M. R. Biointerface Structural Effects on the Properties and Applications of Bioinspired Peptide-Based Nanomaterials. Chem. Rev. 2017, 117, 12641-12704.

3. Karim, R.; Lepeltier, E.; Esnault, L.; Pigeon, P.; Lemaire, L.; Lépinoux-Chambaud, C.;

Clere, N.; Jaouen, G.; Eyer, J.; Piel, G. Enhanced and Preferential Internalization of Lipid Nanocapsules into Human Glioblastoma Cells: Effect of a Surface-Functionalizing NFL Peptide. Nanoscale 2018, 10, 13485-13501.

4. Kojima, S.; Nagata, F.; Kugimiya, S.; Kato, K. Synthesis of Peptide-Containing Calcium Phosphate Nanoparticles Exhibiting Highly Selective Adsorption of Various Proteins. Appl. Surf. Sci. 2018, 458, 438-445.

5. Boge, L.; Hallstensson, K.; Ringstad, L.; Johansson, J.; Andersson, T.; Davoudi, M.; Larsson, P. T.; Mahlapuu, M.; Håkansson, J.; Andersson, M. Cubosomes for Topical Delivery of the Antimicrobial Peptide LL-37. Eur. J. Pharm. Biopharm. 2019, 134, 60-67. 
6. Quan, X.; Sun, D.; Zhou, J. Molecular Mechanism of HIV-1 TAT Peptide and Its Conjugated Gold Nanoparticles Translocating Across Lipid Membranes. Phys. Chem. Chem. Phys. 2019, 21, 10300-10310.

7. Demir, B.; Yilmaz, T.; Guler, E.; Gumus, Z. P.; Akbulut, H.; Aldemir, E.; Coskunol, H.; Colak, D. G.; Cianga, I.; Yamada, S. Polypeptide with Electroactive Endgroups as Sensing Platform for the Abused Drug 'Methamphetamine' by Bioelectrochemical Method. Talanta 2016, 161, 789-796.

8. Liang, P.; Li, Q.; Wu, Z.; Jiang, J.-H.; Yu, R.-Q. Graphene Oxide-Peptide Nanoassembly as a General Approach for Monitoring the Activity of Histone Deacetylases. Analyst 2016, 141, 3989-3992.

9. Chen, J.; Nugen, S. R. Detection of Protease and Engineered Phage-Infected Bacteria Using Peptide-Graphene Oxide Nanosensors. Anal. Bioanal. Chem. 2019, 411, 2487-2492.

10. Povimonsky, A. G.; Rapaport, H. Peptide Coating Applied on the Spot Improves Osseointegration of Titanium Implants. J. Mater. Chem. B 2017, 5, 2096-2105.

11. Yang, Y.; Yang, B.; Li, M.; Wang, Y.; Yang, X.; Li, J. Salivary Acquired PellicleInspired DpSpSEEKC Peptide for the Restoration of Demineralized Tooth Enamel. Biomed. Mater. 2017, 12, 025007.

12. He, J.; Chen, J.; Hu, G.; Wang, L.; Zheng, J.; Zhan, J.; Zhu, Y.; Zhong, C.; Shi, X.; Liu, S. Immobilization of an Antimicrobial Peptide on Silicon Surface with Stable Activity by Click Chemistry. J. Mater. Chem. B 2018, 6, 68-74. 
13. Huq, N. L.; Cross, K. J.; Myroforidis, H.; Stanton, D. P.; Chen, Y.-Y.; Ward, B. R.; Reynolds, E. C. In Biomineralization: From Molecular and Nano-structural Analyses to Environmental Science; Endo, K.; Kogure, T.; Nagasawa, H., Eds.; Springer Nature Singapore: Singapore, 2018; pp 229-239.

14. Mas-Moruno, C. In Peptides and Proteins as Biomaterials for Tissue Regeneration and Repair; Barbosa, M. A.; Martins, M. C. L., Eds.; Elsevier: Duxford, U.K., 2018; pp 73-100.

15. Ortiz-Hernandez, M.; Rappe, K.; Molmeneu, M.; Mas-Moruno, C.; Guillem-Marti, J.; Punset, M.; Caparros, C.; Calero, J.; Franch, J.; Fernandez-Fairen, M. Two Different Strategies to Enhance Osseointegration in Porous Titanium: Inorganic Thermo-Chemical Treatment Versus Organic Coating by Peptide Adsorption. Int. J. Mol. Sci. 2018, 19, 2574.

16. Docter, D.; Westmeier, D.; Markiewicz, M.; Stolte, S.; Knauer, S. K.; Stauber, R. H. The Nanoparticle Biomolecule Corona: Lessons Learned-Challenge Accepted? Chem. Soc. Rev. 2015, 44, 6094-6121.

17. Trindade, R.; Albrektsson, T.; Tengvall, P.; Wennerberg, A. Foreign Body Reaction to Biomaterials: On Mechanisms for Buildup and Breakdown of Osseointegration. Clin. Implant Dent. Relat. Res. 2016, 18, 192-203.

18. Inoue, Y.; Onodera, Y.; Ishihara, K. Initial Cell Adhesion onto a Phospholipid Polymer Brush Surface Modified with a Terminal Cell Adhesion Peptide. ACS Appl. Mater. Interfaces 2018, 10, 15250-15257. 
19. Bellucci, L.; Ardèvol, A.; Parrinello, M.; Lutz, H.; Lu, H.; Weidner, T.; Corni, S. The Interaction with Gold Suppresses Fiber-Like Conformations of the Amyloid $\beta$ (16-22) Peptide. Nanoscale 2016, 8, 8737-8748.

20. Liu, L.; Li, Q.; Zhang, S.; Wang, X.; Hoffmann, S. V.; Li, J.; Liu, Z.; Besenbacher, F.; Dong, M. Identification of a Novel Parallel $\beta$-Strand Conformation within Molecular Monolayer of Amyloid Peptide. Adv. Sci. 2016, 3, 1500369.

21. Bellaiche, M. M. J.; Best, R. B. Molecular Determinants of A $\beta 42$ Adsorption to Amyloid Fibril Surfaces. J. Phys. Chem. Lett. 2018, 9, 6437-6443.

22. Rabe, M.; Verdes, D.; Seeger, S. Understanding Protein Adsorption Phenomena at Solid Surfaces. Adv. Colloid Interface Sci. 2011, 162, 87-106.

23. Heinz, H.; Ramezani-Dakhel, H. Simulations of Inorganic-Bioorganic Interfaces to Discover New Materials: Insights, Comparisons to Experiment, Challenges, and Opportunities. Chem. Soc. Rev. 2016, 45, 412-448.

24. Ozboyaci, M.; Kokh, D. B.; Corni, S.; Wade, R. C. Modeling and Simulation of ProteinSurface Interactions: Achievements and Challenges. Q. Rev. Biophys. 2016, 49, e4.

25. Ramakrishnan, S. K.; Zhu, J.; Gergely, C. Organic-Inorganic Interface Simulation for New Material Discoveries. Wiley Interdiscip. Rev. Comput. Mol. Sci. 2017, 7, e1277.

26. Latour, R. A. Molecular Simulation of Protein-Surface Interactions: Benefits, Problems, Solutions, and Future Directions (Review). Biointerphases 2008, 3, FC2-FC12. 
27. Zhang, L.; Sun, Y. Charged Surface Regulates the Molecular Interactions of Electrostatically Repulsive Peptides by Inducing Oriented Alignment. Langmuir 2018, 34, 43904397.

28. Ross-Naylor, J. A.; Mijajlovic, M.; Zhang, H.; Biggs, M. J. Characterizing the Switching Transitions of an Adsorbed Peptide by Mapping the Potential Energy Surface. J. Phys. Chem. B 2017, 121, 11455-11464.

29. Walsh, T. R. Pathways to Structure-Property Relationships of Peptide-Materials Interfaces: Challenges in Predicting Molecular Structures. Acc. Chem. Res. 2017, 50, 1617-1624.

30. Frenkel, D.; Smit, B. Understanding Molecular Simulation: From Algorithms to Applications; 2nd ed.; Academic Press: San Diego, CA, U.S.A., 2002.

31. Berry, R. S. Potential Surfaces and Dynamics: What Clusters Tell Us. Chem. Rev. 1993, 93, 2379-2394.

32. Wales, D. J. Energy Landscapes: Applications to Clusters, Biomolecules and Glasses; Cambridge University Press: Cambridge, U.K., 2003.

33. Wales, D. J.; Bogdan, T. V. Potential Energy and Free Energy Landscapes. J. Phys. Chem. B 2006, 110, 20765-20776.

34. Wales, D. J. Exploring Energy Landscapes. Annu. Rev. Phys. Chem. 2018, 69, 401-425.

35. Wales, D. J. Discrete Path Sampling. Mol. Phys. 2002, 100, 3285-3305. 
36. Ross-Naylor, J. A.; Mijajlovic, M.; Biggs, M. J. Energy Landscape Mapping and Replica Exchange Molecular Dynamics of an Adsorbed Peptide. J. Phys. Chem. B, submitted for publication, 2020.

37. Huang, J.; Rauscher, S.; Nawrocki, G.; Ran, T.; Feig, M.; de Groot, B. L.; Grubmuller, H.; MacKerell, A. D., Jr. CHARMM36m: An Improved Force Field for Folded and Intrinsically Disordered Proteins. Nat. Methods 2017, 14, 71-73.

38. Małolepsza, E.; Strodel, B.; Khalili, M.; Trygubenko, S.; Fejer, S. N.; Wales, D. J. Symmetrization of the AMBER and CHARMM Force Fields. J. Comput. Chem. 2010, 31, 14021409.

39. Braun, R.; Sarikaya, M.; Schulten, K. Genetically Engineered Gold-Binding Polypeptides: Structure Prediction and Molecular Dynamics. J. Biomater. Sci. Polym. Ed. 2002, $13,747-757$.

40. Steele, W. A. The Physical Interaction of Gases with Crystalline Solids: I. Gas-Solid Energies and Properties of Isolated Adsorbed Atoms. Surf. Sci. 1973, 36, 317-352.

41. Trucano, P.; Chen, R. Structure of Graphite by Neutron-Diffraction. Nature 1975, 258, 136-137.

42. Wales, D. J.; Doye, J. P. K. Global Optimization by Basin-Hopping and the Lowest Energy Structures of Lennard-Jones Clusters Containing up to 110 Atoms. J. Phys. Chem. A 1997, 101, 5111-5116.

43. Liu, D. C.; Nocedal, J. On the Limited Memory BFGS Method for Large Scale Optimization. Math. Prog. 1989, 45, 503-528. 
44. Munro, L. J.; Wales, D. J. Defect Migration in Crystalline Silicon. Phys. Rev. B: Condens. Matter Mater. Phys. 1999, 59, 3969-3980.

45. Becker, O. M.; Karplus, M. The Topology of Multidimensional Potential Energy Surfaces: Theory and Application to Peptide Structure and Kinetics. J. Chem. Phys. 1997, 106, 1495-1517.

46. Dijkstra, E. W. A Note on Two Problems in Connexion with Graphs. Numer. Math. 1959, 1, 269-271.

47. Carr, J. M.; Trygubenko, S. A.; Wales, D. J. Finding Pathways Between Distant Local Minima. J. Chem. Phys. 2005, 122, 234903.

48. Evans, D. A.; Wales, D. J. Folding of the GB1 Hairpin Peptide from Discrete Path Sampling. J. Chem. Phys. 2004, 121, 1080-1090.

49. Carr, J. M.; Wales, D. J. Global Optimization and Folding Pathways of Selected $\alpha$ Helical Proteins. J. Chem. Phys. 2005, 123, 234901.

50. Trygubenko, S. A.; Wales, D. J. A Doubly Nudged Elastic Band Method for Finding Transition States. J. Chem. Phys. 2004, 120, 2082-2094.

51. Carr, J. M.; Wales, D. J. In Latest Advances in Atomic Cluster Collisions: Structure and Dynamics from the Nuclear to the Biological Scale; Connerade, J.-P.; Solov'yov, A., Eds.; Imperial College Press: London, U.K., 2008; pp 321-330.

52. Stillinger, F. H.; Weber, T. A. Packing Structures and Transitions in Liquids and Solids. Science 1984, 225, 983-989. 
53. Strodel, B.; Whittleston, C. S.; Wales, D. J. Thermodynamics and Kinetics of Aggregation for the GNNQQNY Peptide. J. Am. Chem. Soc. 2007, 129, 16005-16014.

54. Evans, D. A.; Wales, D. J. Free Energy Landscapes of Model Peptides and Proteins. J. Chem. Phys. 2003, 118, 3891-3897.

55. Wales, D. J. Decoding Heat Capacity Features from the Energy Landscape. Phys. Rev. E: Stat., Nonlinear, Soft Matter Phys. 2017, 95, 030105.

56. Wales Group Home Page. https://www.ch.cam.ac.uk/group/wales/ (accessed October 16, 2019).

57. Brooks, B. R.; Brooks, C. L., III; MacKerell, A. D., Jr.; Nilsson, L.; Petrella, R. J.; Roux, B.; Won, Y.; Archontis, G.; Bartels, C.; Boresch, S., et al. CHARMM: The Biomolecular Simulation Program. J. Comput. Chem. 2009, 30, 1545-1614.

58. Humphrey, W.; Dalke, A.; Schulten, K. VMD: Visual Molecular Dynamics. J. Mol. Graphics 1996, 14, 33-38.

59. Joseph, J. A.; Wales, D. J. Intrinsically Disordered Landscapes for Human CD4 Receptor Peptide. J. Phys. Chem. B 2018, 122, 11906-11921.

60. Neelamraju, S.; Gosavi, S.; Wales, D. J. Energy Landscape of the Designed Protein Top7. J. Phys. Chem. B 2018, 122, 12282-12291.

61. Röder, K.; Wales, D. J. Energy Landscapes for the Aggregation of Aß17-42. J. Am. Chem. Soc. 2018, 140, 4018-4027. 
62. Wales, D. J.; Disney, M. D.; Yildirim, I. Computational Investigation of RNA A-Bulges Related to the Microtubule-Associated Protein Tau Causing Frontotemporal Dementia and Parkinsonism. J. Phys. Chem. B 2019, 123, 57-65.

63. Wright, L. B.; Walsh, T. R. Efficient Conformational Sampling of Peptides Adsorbed onto Inorganic Surfaces: Insights from a Quartz Binding Peptide. Phys. Chem. Chem. Phys. 2013, 15, 4715-4726.

64. Röder, K.; Joseph, J. A.; Husic, B. E.; Wales, D. J. Energy Landscapes for Proteins: From Single Funnels to Multifunctional Systems. Adv. Theory Simul. 2019, 2, 1800175.

65. Smiatek, J.; Heuer, A. Calculation of Free Energy Landscapes: A Histogram Reweighted Metadynamics Approach. J. Comput. Chem. 2011, 32, 2084-2096.

66. Banerjee, R.; Cukier, R. I. Transition Paths of Met-Enkephalin from Markov State Modeling of a Molecular Dynamics Trajectory. J. Phys. Chem. B 2014, 118, 2883-2895.

67. Mijajlovic, M.; Penna, M. J.; Biggs, M. J. Free Energy of Adsorption of Proteins at Fluid/Solid Interfaces Using Molecular Simulation. In Chemeca 2011: Engineering a Better World, Engineers Australia: Sydney Hilton Hotel, NSW, Australia, 2011; pp 1221-1231. 
TOC GRAPHIC

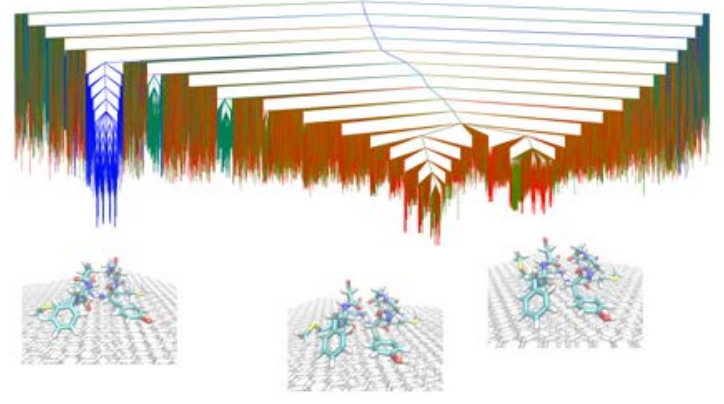

